\title{
L'AUTORÉGULATION PRAGMATIQUE DU JOURNALISME EN AMÉRIQUE DU NORD
}

\author{
Marc-François Bernier ${ }^{1}$
}

\section{Remarques préliminaires}

Les journalistes savent, pour la plupart d'entre eux, que leurs pratiques ne sont légitimes qu'à l'intérieur de certains paramètres, les principaux étant l'intégrité, l'honnêteté et le service du public qu'ils affirment représenter.

Mais les pressions d'ordre politique, économique, social, affectif et idéologique influencent quotidiennement les pratiques journalistiques et ce n'est pas sans dépit que la profession s'est peu à peu résignée à se doter de règles déontologiques conformes aux principes éthiques et valeurs qui fondent à la fois leur légitimité sociale, leurs droits constitutionnels et leur rhétorique professionnelle. C'est à cette triple source que les journalistes puisent les arguments qu'ils servent par la suite à ceux qui voudraient les contrôler en insistant trop sur leurs responsabilités, au détriment de leurs libertés.

1 Docteur en science politique, journaliste au Journal de Québec.

Recherches en communication, $\mathrm{n}^{\circ}$ 9, (1998). 
En même temps qu'ils se défendent contre les incursions d'agents extérieurs voulant contrôler davantage leurs pratiques, les journalistes soucieux de leurs droits et devoirs d'informer n'ignorent pas la nécessité de veiller eux-mêmes à endiguer les dérives et à freiner les dérapages qui résultent des impératifs économiques des entreprises de presse, des contraintes de temps qui s'imposent de plus en plus avec la télédiffusion en direct d'événements majeurs et la mise à jour de sites Internet, quand ils ne sont pas attribuables à la poursuite de la notoriété immédiate et monnayable.

L'autorégulation des pratiques journalistiques en Amérique du Nord a essentiellement deux sources d'influence qui convergent souvent, mais s'opposent parfois. Il y a d'abord le filon éthique ou philosophique qui a émergé depuis le début du siècle aux États-Unis, mais a rapidement gagné le Canada anglais et ses entreprises de presse privées et publiques. Isolé de cette tendance par sa culture française et une religiosité particulière qui n'a heureusement plus cours, le Québec a résisté longtemps avant de s'inspirer du courant anglo-saxon. Ce courant insiste pour que la profession soit libre de tout entrave et assume elle-même, sur une base volontaire, le mandat de surveillance des pratiques professionnelles. L'autorégulation est alors un moyen d'échapper aux pressions externes (juridique, économique, étatique, idéologique ou religieuse) qui sont perçues comme d'inacceptables tentatives de censure à l'égard de la liberté de presse.

Il y a aussi une source légale. Dans des pays dotés de constitutions et de chartes privilégiant les droits individuels et la liberté d'expression, la tension est permanente entre ces droits qui opposent souvent liberté de la presse et droits des citoyens à la vie privée ou à la réputation. $\mathrm{Il}$ y a donc intérêt à ne pas abuser d'une liberté pouvant contrevenir aux droits d'individus en mesure de faire payer chèrement leur négation de la part des médias d'information. Cela est d'autant plus vrai depuis quelques années, alors que les médias sont sévèrement jugés par les tribunaux et le public qui les perçoivent souvent comme des entraves au bon fonctionnement de la société. Une façon de contenir les foudres des tribunaux est sans doute de se doter de règles déontologiques qui délimitent tant bien que mal les pratiques acceptables de celles qui risquent de léser le public et de générer des poursuites devant les tribunaux.

A en croire la multitude de poursuites devant les tribunaux aussi bien aux États-Unis qu'au Canada et au Québec, il semble que 
l'implantation de mécanismes d'autorégulation n'est ni suffisante ni satisfaisante pour tous. Il faut cependant faire remarquer que bien des entreprises de presse n'ont aucun code de déontologie, encore moins d'ombudsman. D'autres, tel le New York Times, ont farouchement lutté contre la création de conseils de presse. Au Québec, ce sont les médias d'information de l'empire Québécor qui refusent de contribuer au financement du Conseil de presse fondé en 1973. Bref, l'autorégulation ne touche qu'une partie des médias d'information et chacun y accorde plus ou moins d'importance, selon les enjeux du moment. Cela ne suffit pas à empêcher les dérapages médiatiques dont l'ampleur échappe de moins en moins au regard des citoyens et des tribunaux.

Du reste, les enquêtes scientifiques et les sondages qui cherchent à connaître l'opinion des publics relativement aux médias d'information font constamment état du mécontentement des citoyens, même si dans bien des cas ce mécontentement est dû à des décisions éditoriales et non à des carences déontologiques ou éthiques ${ }^{1}$. Le public américain désire néanmoins que les entreprises de presse se dotent de moyens d'échanger avec le public sur des questions d'équité et d'éthique professionnelle. Il souhaite aussi que la fonction de service public des entreprises de presse passe avant la recherche de profits. L'enquête révèle aussi que $58 \%$ des répondants croient que les journalistes sont plus intéressés à faire valoir leurs opinions dans leurs comptes rendus qu'à faire du travail équitable et impartial, et $57 \%$ des citoyens $(72 \%$ des politiciens et $38 \%$ des journalistes interrogés) croient que les journalistes ne sont pas plus honnêtes que les politiciens qu'ils critiquent.

Un sondage réalisé en 1994 pour le Forum Magazine² révélait notamment que le public reproche l'insensibilité des journalistes face à la douleur des victimes d'événements malheureux (82\% des répondants), et la trop grande importance accordée à la vie privée des élus $(75 \%)$. L'abus du recours aux sources anonymes est critiqué par

1 B. PhILLIPS et B. Kees, Nothing Sacred - Journalism, Politics and Public Trust in a Tell-All Age, Freedom Forum center, 1995, document internet, pagination personnelle, [http//: www.fac.org/publicat/nothsacr/nstoc.htm].

2 "The love-hate relationship between politicians and the news media", The Forum Magazine, septembre 1994, document internet, p. 3. 
$58 \%$ des répondants ${ }^{1}$. Peter Desbarats ${ }^{2}$ évoque quant à lui un sondage réalisé en 1987 selon lequel la majorité de la population canadienne estime que les médias font un effort conscient afin d'influencer ou de biaiser l'opinion publique. Près de la moitié des répondants estimaient que le biais des médias constituait un problème sérieux $(12 \%)$ ou assez sérieux $(37 \%)$ et près du tiers $(31 \%)$ des répondants indiquaient qu'ils supporteraient une réglementation gouvernementale visant à garder les médias honnêtes et objectifs. Cependant, Levine cite un sondage Macleans/Decima de novembre $1990^{3}$ selon lequel une majorité de Canadiens considéraient comme assez rigoureuse la couverture médiatique des événements, croyaient que les médias les aidaient à comprendre ces événements et qu'ils n'étaient pas responsables du cynisme présent au Canada. L'humeur du public serait donc, elle aussi, matière à recherche afin de mieux comprendre certaines contradictions apparentes.

Ce que cette vague de mécontentement nous indique, c'est que l'autorégulation ne saurait être qu'un procédé superficiel, une tactique visant simplement à se donner bonne conscience. Le public doit avoir des raisons objectives de croire que les médias sont en mesure de se prendre en main et de le protéger contre les excès d'une minorité de journalistes douteux. Une telle conviction se refléterait sans doute sur l'appréciation générale des citoyens au sujet des journalistes.

\section{Survol des principaux mécanismes d'autorégulation en Amérique du Nord}

Dans la présente section, je m'appliquerai à évoquer certains avantages et certaines limites, certains espoirs et certaines craintes reliées aux principaux mécanismes d'autorégulation. Ces propos éclaireront mieux les sections trois et quatre qui constituent une réflexion et une approche plus personnelle de ces questions.

1 P. Prichard, "News junkies, News critics ; How Americans use the news and what they think about it", The Freedom Forum, 1997, document internet.

2 P. Desbarats, Guide to Canadian News Media, Toronto, Harcourt Brace Jovanovich, 1990, p. 123.

3 A. Levine, Scrum Wars : The Prime Ministers and the Media, Toronto \& Oxford, Dundurn Press, 1993, p. 359. 


\subsection{Conseils de presse}

Il n'y a que quelques conseils de presse en Amérique du Nord. Aux États-Unis, le plus connu est certes celui du Minnesota. Au Canada, on en retrouve dans plusieurs provinces (Colombie Britannique, Provinces Maritimes ${ }^{1}$, Ontario et Québec). Une tentative plus récente de créer un conseil de presse dans l'État du Kentucky a été un échec parce que cela impliquait une mise de fonds de un million de dollars américains ${ }^{2}$. On connaît aussi la triste fin du National News Council des États-Unis, fondé en 1973 et aboli en 1984 faute de ressources financières et de soutiens majeurs de la part de médias tels le New York Times, le Chicago Tribune et le réseau de télévision NBC. Certains auteurs, dont Desbarats, sont d'avis qu'une des faiblesses du NNC a été sa timidité à émettre des commentaires critiques à propos des grands propriétaires de médias, conjuguée à un manque de notoriété ${ }^{3}$. Au Canada, il faut noter que si le Conseil de presse du Québec est constamment à court de moyens financiers, il jouit d'un appui certain chez les patrons de la presse qui y siègent, tandis que celui de l'Ontario a vu son membership passer de 10 à 35 membres de 1982 à 1983, alors que la question de la concentration de la presse préoccupait le gouvernement fédéral ${ }^{4}$.

Bien que des représentants du public y soient souvent présents, on peut considérer que les conseils de presse sont une forme d'autorégulation lorsqu'ils résultent d'une initiative volontaire des milieux de la presse face à des menaces réelles ou appréhendées d'intervention étatiques, ce qui a été le cas au Canada ${ }^{5}$.

Malheureusement, les conseils de presse souffrent d'un réel manque de visibilité, quand ce n'est pas d'une faible autorité morale auprès du public comme des journalistes et des entreprises de presse. Selon l'ex-directrice du quotidien Le Devoir, Lise Bissonnette, le CPQ a "démontré les limites de la bonne volonté. Ses jugements n'ont qu'une influence très limitée, quand ils ne sont pas bafoués et tournés

1 Nouveau Brunswick, Terre Neuve, Ile du Prince-Édouard et Nouvelle Écosse.

2 D. BoeYINK, "Public Understanding, Professional Ethics, and the News : A Response to Jane Rhodes", Federal Communications Law Journal, vol. 47, $\mathrm{n}^{\circ} 1$, octobre 1994, document internet, p. 1.

3 P. Desbarats, op. cit., p. 169.

4 Ibid., p. 163.

5 D. PRICHARD, "The Role of Press Councils in a System of Media Accountability : The Case of Quebec", Canadian Journal of Communication, vol. 16, 1991, p. 7677. 
en dérision par ceux qu'ils visent le plus souvent"1. Mais il faut signaler certaines considérations des opposants aux conseils de presse. Selon une étude, la majorité des gestionnaires de presse des ÉtatsUnis craignent que les individus aient recours aux conseils de presse afin d'attirer sur eux l'attention du public, et ce aux dépens des médias en cause ${ }^{2}$. Bien entendu, ils sont nombreux à craindre que ces conseils attaquent la liberté de la presse et menacent en quelque sorte le principe de l'indépendance des journalistes ${ }^{3}$. D'autres craignent également que les conseils en viennent, un jour ou l'autre, à se lancer dans la chasse aux sorcières, comme l'a constaté le journaliste Casey Bukro quand il a commencé à faire la promotion de conseils de presse locaux aux États-Unis ${ }^{4}$. D'autres ont peur de voir les normes, les règles de conduite et même les décisions des conseils de presse être reprises par les tribunaux dans des jugements impliquant des entreprises de presse.

De façon encore plus fondamentale, il y a une objection qui porte sur la manière dont certains conseils de presse abordent les questions de déontologie, soit le mode jurisprudentiel qui représente "une rupture radicale entre l'éthique et la déontologie. En effet, les décisions... si justes qu'elles puissent sembler, obéissent à la technique des précédents d'après laquelle on inférerait d'une série de décisions, cas par cas, un certain nombre de principes. Or, cette approche est étrangère à l'éthique et à la morale, puisque l'on doit attendre de ces dernières qu'elles prédéterminent des décisions, plutôt que d'en résulter"'. D'autres chercheurs ont aussi mis en évidence la faiblesse des conseils de presse jurisprudentiels, tel le Conseil de presse du Québec, qui n'ont pas réussi à mettre systématiquement à jour les principes et valeurs qui sous-tendent leurs décisions et font preuve d'impressionnisme, de confusion ou de contradictions dans leurs jugements ${ }^{6}$.

1 L. BissonnetTe, "L'invasion de la vie privée : une complicité active", Le 30, vol. $16, \mathrm{n}^{\circ} 4$, mai 1992 , p. 9.

2 R. SCHAFER, "News Media and Complainant Attitudes Toward the Minnesota Press Council", Journalism Quarterly, n 56, hiver 1979, p. 745.

3 D. BoEYINK, "Public Understanding...", op. cit., p. 1.

4 E. Goodwn, Groping for Ethics in Journalism, Iowa State University Press/Ames, 1986, second edition, p. 15.

5 G. Giroux, "La déontologie professionnelle dans le champ du journalisme. Portée et limites", Communication, vol. 12, n 2, Automne 1991, p. 123-124.

6 D. PrITCHARD, "The Role of Press Councils...", op. cit., p. 76. U. Deschênes, L'insoutenable légèreté du discours. L'analyse de la jurisprudence du Conseil de 


\subsection{Ombudsman}

Les ombudsmen constituent une autre réponse de la profession à la nécessité de prendre en charge la surveillance de ses dérapages, mais ces acteurs sont encore très peu nombreux. Aux États-Unis, ils sont seulement 34 alors qu'on retrouve 1500 quotidiens, et ils ne sont que quelques-uns au Canada, dont ceux des réseaux anglais et français de la Société Radio-Canada. Le quotidien anglophone de Montréal, The Gazette, en avait un mais il n'a pas été remplacé depuis plus d'un an. Selon l'ONO (Organization of News Ombudsmen), il y aurait 96 ombudsmen dans 14 pays, dont quatre au Canada (incluant les deux de la Société Radio-Canada/Canadian Broadcasting Corporation).

Le rôle de l'ombudsman ne fait pas l'unanimité. Pour certains, sa fonction de chien de garde devrait plutôt être assumée par les gestionnaires des salles de rédaction, rapporte David Shaw du Los Angeles Times ${ }^{1}$. D'autres les accusent d'être des critiques superficiels à la solde des organisations qui les emploient et leur imposent des limites à ne pas franchir. On a ainsi accusé Richard Harwood, premier ombudsman du Washington Post en 1970, d'être un tiède critique du Post et, surtout, de se porter à la défense de la direction de ce quotidien ${ }^{2}$.

On lui reproche aussi d'intervenir après le dérapage déontologique au lieu de le prévenir, quand ce n'est pas de s'en tenir généralement à expliquer aux lecteurs la politique du journal qu'ils n'ont pas le pouvoir de modifier ${ }^{3}$.

En tenant compte du nombre restreint d'ombudsmen ainsi que des limites de leur pouvoir, on peut partager l'avis de Boeyink selon qui le recours aux ombudsmen a eu un impact marginal au niveau de l'imputabilité 4 des médias d'information, surtout du fait que ce poste implique des coûts importants qui ne peuvent être assumés par tous

presse du Québec, mémoire de maîtrise, Département d'information et de communication, Faculté des Arts, Université Laval, 1996, 101 pages.

1 D. SHAW, Press Watch: A Provocative Look at How Newspapers Report the News, New York, MacMillan, 1984, p. 13.

2 M. LeE. et N. Solomon, Unreliable Sources : A Guide to Detecting Bias in News Media, New York, Carol Publishing Group, 1990, p. 21.

3 C. DORNAN, "Libre de rendre des comptes : La responsabilité de la presse écrite", in F. FLETCHER, Sous l'œil des journalistes. La couverture des élections au Canada, Commission royale sur la réforme électorale et le financement des partis politiques, Montréal, Wilson \& Lafleur Limitée, 1991, vol. 22, p. 171.

4 A prendre dans le sens de "accountability". 
les journaux. Il ajoute que plusieurs des ombudsmen en fonction aux États-Unis en 1994 étaient davantage des chroniqueurs des plaintes du public que de véritables défenseurs des lecteurs ou de vrais critiques des médias ${ }^{1}$. Desbarats rapporte une étude américaine menée auprès de 32 ombdusmen selon qui les dirigeants ne veulent pas avoir un ombudsman puissant qui s'exprimerait au nom des lecteurs car cela pourrait affecter le moral des troupes, mais ils ne veulent pas non plus d'ombudsman faible exprimant seulement le point de vue de l'entreprise, car cela ne serait qu'un simple exercice de relations publiques. Desbarats fait aussi état d'une enquête canadienne réalisée en 1986 auprès de journalistes du Toronto Star et du quotidien montréalais The Gazette qui a montré que la fonction d'ombudsman suscite des réactions différentes parmi les journalistes. Ceux de la Gazette (46\%) estimaient que l'ombudsman n'était pas très utile dans leur pratique quotidienne contre $35 \%$ au Star. La moitié des journalistes de la Gazette étaient d'accord pour dire que l'ombudsman était incapable de vraie indépendance contre $28 \%$ au Star. Dans les deux journaux, quatre journalistes sur cinq étaient d'accord pour dire que l'ombudsman était utile pour les lecteurs, mais ils étaient $67 \%$ au Star et $32 \%$ à la Gazette à dire qu'il pouvait leur être utile ${ }^{2}$. Il faut aussi signaler des cas où l'ombudsman a eu maille à partir avec son employeur : dans un cas, l'ombudsman a préféré démissionner plutôt que de soumettre son avis à ses supérieurs avant publication, dans l'autre cas l'ombudsman croit que l'élimination de sa fonction est due à son activisme en faveur du public ${ }^{3}$.

L'ombudsman, qui assume une fonction de contrôle de la qualité de l'information, peut pourtant exercer une influence positive sur les journalistes, surtout auprès des jeunes. On peut aussi le considérer comme une personne ressource supervisant le travail journalistique, en espérant que son intervention préviendra le journalisme erratique ou frivole qui constituent des menaces à long terme pour la presse et la démocratie ${ }^{4}$. En ce sens, il pourrait instaurer dans la salle de rédaction une tradition de dialogue portant sur l'éthique et la

1 D. BOEYINK, "Public Understanding...", op. cit., p. 2.

2 P. DESBARATS, op. cit., p. 171 .

3 W. BRIDGES \& J. BRIDGES. "Newspaper ombudsman's role during a presidential campaign", Newspaper Research Journal, Vol. 16, $\mathrm{n}^{\circ} 2,1995$, p. 2, (http://www.facsnet.org/cgi-bin/nrj_search.cgi?row=6).

4 P. Desbarats, op. cit., p. 123. 
déontologie du journalisme ${ }^{1}$. En permettant au public de faire connaître ses doléances, et s'il enquête sérieusement à leur sujet, il ne fait pas de doute que l'ombudsman contribue positivement à l'imputabilité recherchée par tout mécanisme d'autorégulation crédible.

Je ne veux pas passer sous silence le rôle positif que certains ombudsmen ont eu à la suite de la campagne électorale présidentielle de 1992. Une étude réalisée aux États-Unis ${ }^{2}$ révèle que les plaintes du public ont été prises en compte quand elles étaient considérées comme légitimes. Par exemple, certains journaux ont accordé une plus grande attention à l'espace accordé aux différents candidats, un autre a augmenté l'espace réservé aux commentaires des lecteurs, un autre a publié une grille horaire des présences des candidats à la télévision, un autre s'est montré plus attentif aux titres tandis qu'un dernier journal a retiré un chroniqueur de sa première page après les élections ${ }^{3}$. Les auteurs de la recherche sont d'avis que si les ombudsmen n'ont pas le pouvoir de changer eux-mêmes les choses, ils ont une influence certaine pouvant induire des changements dans la couverture des événements et la présentation des informations. Ces changements étant en faveur de l'équité et de l'équilibre dans la couverture.

\subsection{Métajournalisme}

Le métajournalisme constitue peut-être la voie autorégulatrice la plus en vogue actuellement. Je nomme métajournalisme ce que les anglo-saxons appellent media critics. Il s'agit essentiellement d'aborder de manière journalistique la question des pratiques des journalistes et des entreprises de presse. Tout y passe : conflit d'intérêts, procédés clandestins, manque de rigueur des informations diffusées, non respect de la vie privée, etc. Dans cette dynamique, les médias rapportent de plus en plus les dérapages de leurs concurrents et font souvent une autocritique quand la situation l'exige. De plus, on fait constamment référence aux attentes du public, présupposées ou documentées, afin de légitimer la critique exercée. Cette façon de

1 M.-Fr. BERNIER, Les planqués : le journalisme victime des journalistes, Montréal, VLB, 1995, p. 160.

2 L. BRIDGES \& J. BRIDGES, op. cit.

3 Ibid., p. 9. 
procéder rend d'autant plus légitime le métajournalisme que celui-ci doit affronter méfiance et vives contestations au sein d'une profession qui a tendance à qualifier de "chasseurs de sorcières" ceux qui exercent à son endroit la même vigilance qu'exercent les journalistes à propos d'autres institutions sociales publiques, privées et communautaires. On sait que les réflexes journalistiques sont souvent valorisés en Amérique du Nord, mais que la réflexion et l'autoréflexion critique sont des attitudes intellectuelles considérées avec suspicion, sans doute parce qu'on les assimile aux critiques provenant d'individus et de groupes qui cherchent parfois à museler la presse. Chez les journalistes, on a quelque peine à admettre que certaines récriminations provenant de sources aux motivations douteuses puissent néanmoins être fondées. Selon Bacon, les membres du "4e pouvoir" ignorent souvent ou discréditent les critiques externes, sans égard à leur validité, en invoquant les protections constitutionnelles, avec arrogance parfois. Lorsque les critiques proviennent de la profession, les réactions sont différentes car les commentaires sont plus difficiles à écarter ou ignorer ${ }^{1}$.

Au Los Angeles Times, David Shaw exerce sa fonction de critique des médias depuis 1974 et son patron de l'époque, William Thomas, l'avait averti de se trouver des amis en dehors de la profession, car il perdrait rapidement ceux qu'il y avait. Une enquête réalisée en 1995 a révélé que des collègues de Shaw ont eu à son endroit des réactions que Bacon qualifie d'hystériques, certains refusant même de collaborer à son travail en rejetant les demandes $d^{\prime}$ 'entrevue ${ }^{2}$. Je me permets ici une note plus personnelle en ajoutant qu'à titre de critique du travail journalistique au Québec j'ai aussi dû subir les foudres de mes pairs, collègues aussi bien qu'employeur. Les courriéristes parlementaires de l'Assemblée nationale du Québec ont même déjà manœuvré pour m'empêcher de prononcer une conférence lors d'un colloque sur les médias et les parlementaires, menaçant de boycotter l'événement ${ }^{3}$.

Malgré tout, il ne se passe plus un grand événement médiatisé qui ne donne prise à l'analyse et la dénonciation des excès médiatiques, ce qui est considéré comme une bonne chose par

1 M. Bacon, "Criticizing the critics : L.A. Times' David Shaw and his detractors", Newspaper Research Journal, vol. 16, $\mathrm{n}^{\circ}$ 1, winter 1995, p. 22.

2 Ibid., p. 24.

3 N. DELISLE, "Journalistes et politiciens : chambre à part ou concubinage ?", Le 30, vol. $21, \mathrm{n}^{\circ} 1$, décembre-janvier 1997, p. 21. 
certains ${ }^{1}$. Le cas le plus évident de l'année 1998 a certes été l'affaire Lewinsky-Clinton. Mais on a vu aussi ces questionnements lors du décès de Lady Di et il fait maintenant partie de la couverture des grandes campagnes électorales aux États-Unis et au Canada, même si cette autocritique n'est pas encore l'apanage de la majorité des entreprises de presse.

Le métajournalisme aura un effet important sur les pratiques journalistique dans la mesure où ses dénonciations et analyses seront basées sur les règles déontologiques et critères éthiques reconnus, et non sur des grilles d'analyses contaminées par les intérêts partisans, idéologiques et économiques de leurs auteurs.

Le métajournalisme a également ses "vedettes", tels David Shaw du Los Angeles Times, ou Howard Kurtz du Washington Post. Il a son temps d'antenne à CNN, ses sites internet, ses revues de toutes tendances idéologiques ( $C J R, A J R$, Brill's Content, Forbes Media Critic, FAIR, etc.). Au Québec, on a vu ces dernières années les deux grands quotidiens de langue française, La Presse et Le Soleil de Québec expliquer davantage à leurs lecteurs certaines de leurs décisions rédactionnelles. Même si cet exercice d'autocritique est assez timide, il témoigne d'un besoin de rétablir la communication chez ces quotidiens dont le tirage a considérablement diminué depuis plusieurs années, en raison de la concurrence vive de la télévision et des quotidiens populaires de Québécor.

Le métajournalisme doit aussi s'alimenter des résultats de la recherche scientifique. L'analyse sociologique des pratiques journalistiques ou l'évaluation normative de celles-ci ne doivent pas être cantonnées dans les revues savantes. Les scientifiques ont un devoir de service public, également, et ils devraient faciliter la communication des résultats de leurs démarches.

Le métajournalisme a bien entendu ses limites. Par exemple, celle formulée par Alter ${ }^{2}$ selon qui le problème fondamental est que la critique s'exerce en vertu de certaines normes du journalisme, mais que ces mêmes normes ne sont jamais mises en doute. D'autres font valoir que la vigueur d'une telle critique interne est laissée à la merci des propriétaires de médias, notamment dans les journaux où le métajournalisme s'exercerait surtout aux dépens de la production

1 H. KurTz, "Watchdogs Yelp When Made to Heel", Washington Post, 13 juillet 1998, p. D1.

2 J. Alter, "New Questions - That's What Media Critics Need, Media Studies Journal, printemps 1995, vol. $9, \mathrm{n}^{\circ} 2$. 
télévisuelle, rarement à propos de l'écrit ${ }^{1}$. Les éditeurs du Media Studies Journal affirmaient, en 1995, que seulement deux des cinq grands quotidiens nationaux des États-Unis (Los Angeles Times et Washington Post) ont établi une véritable tradition de critique des médias, alors que les magazines auraient un meilleur dossier en matière de commentaires et de reportages sur les médias, les réseaux de télévision arrivant loin derrière ${ }^{2}$. La cablodistribution et la radio, avec ses animateurs parfois démagogues, ont fait des progrès en matière de critique des médias, malgré le biais idéologique qu'on peut souvent y déplorer.

Une attaque récurrente à l'endroit du métajournalisme, mais qui est également reprise pour s'opposer à toutes formes d'autorégulation et d'hétérorégulation, consiste à en appeler au verdict du public. La croyance largement répandue chez les journalistes en une infaillibilité du jugement populaire suffit souvent à rejeter toute forme de critique interne ou externe dans la mesure où les tirages et les cotes d'écoute se maintiennent ou augmentent. Néanmoins, lorsque ces indices sont à la baisse, les médias cherchent des solutions liées à la mise en marché ou à la présentation de contenus différents. Rarement a-t-on entendu un gestionnaire affirmer qu'il allait redresser ses ventes ou ses cotes d'écoute en incitant ses journalistes à mieux observer les normes professionnelles reconnues.

En somme, on fait appel au public pour ignorer ces considérations éthiques et déontologiques lorsque les affaires vont bien, mais quand la situation se dégrade, on trouve d'autres excuses pour toujours ignorer ces considérations. Ce qui conduit d'autant plus à croire que les préoccupations liées à la critique des pratiques journalistiques doivent d'abord relever du droit du public à une information de qualité, complète et impartiale, plutôt qu'à gonfler les revenus publicitaires ou de vente, bien que cette dernière éventualité ne soit nullement à dédaigner, bien au contraire. L'impact de ces questions professionnelles sur la situation économique des entreprises de presse pourrait cependant se faire ressentir si la critique de certains

1 A. LeE et N. Solomon, op. cit., p. XIV. D. Graber, "Mass Media et campagnes électorales aux États-Unis», in Fr. FLETCHER (dir.) Médias, élections et démocratie, Montréal, Wilson \& Lafleur, 1991, Commission royale sur la réforme électorale et le financement des partis, vol. 19, p. 164.

2 Media Studies Journal, «Preface», in Media Studies Journal, printemps 1995, vol. $9, \mathrm{n}^{\circ} 2$, p. 2 , préface de l'équipe éditoriale du numéro spécial consacré aux Media Critics. 
médias devient telle que le public, dorénavant informé de ces dérapages, en vient à modifier ses habitudes d'achat et d'écoute afin d'exprimer son mécontentement. C'est peut-être l'hypothèse d'un public bien informé au sujet de ceux qui prétendent l'informer qui alimente les craintes des propriétaires, gestionnaires et praticiens des entreprises de presse, face au métajournalisme.

Du reste, le public nord-américain souhaite de plus en plus être informé sur les pratiques journalistiques ${ }^{1}$. Un sondage réalisé en 1996 auprès de 3000 Américains révèle, par exemple, que $79 \%$ des répondants demandent aux journalistes d'accorder plus de couverture à leurs propres erreurs. Cette opinion est encore plus révélatrice du jugement populaire sur les médias quand on ajoute que $85 \%$ des répondants sont d'accord avec le principe de créer un conseil de presse national et sont favorables à une loi qui imposerait la norme de l'équité (fairness doctrine) afin d'assurer une couverture équilibrée de tous les points de vues relatifs à une controverse ; $70 \%$ sont favorables à des amendes légales pour punir les reportages biaisés ou manquant de rigueur; $53 \%$ supportent l'idée d'obliger les journalistes à détenir un permis pour travailler ; $50 \%$ sont favorables à une réforme de la loi qui faciliterait les poursuites pour les cas de diffamation ${ }^{2}$.

La résistance du milieu médiatique au métajournalisme semble pourtant contraire à l'intérêt public car il "serait certainement profitable à l'ensemble de la population de connaitre certaines des méthodes et des priorités de la presse, les contraintes, organisationnelles ou autres, auxquelles elle est soumise et le rôle politique qu'elle assume en tant que véhicule de l'expression

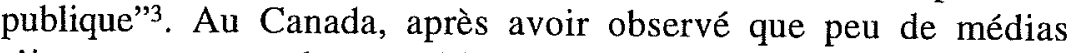
s'interrogent sur leurs méthodes de couverture électorale, des chercheurs ont recommandé au gouvernement canadien d'encourager "l'auto-évaluation des médias, particulièrement en rapport avec les conséquences politiques découlant d'un cynisme accru et d'analyses manquant de profondeur au cours d'une campagne électorale"4.

1 B. PhILlips et B. KeEs, op. cit., p. 2. American Society of Newspaper Editors, Insight on the Values, Journalism Values Institute, vol. 11, 1995.

2 R. Lichter \& L. LiCHTER, "What Do the People Want from the Press ?", Media Monitor, vol. XI, $\mathrm{n}^{\circ}$ 2, mai-juin 1997, numéro spécial, document internet, pp. 6-7, (http:www.cmpa.com/Mediamon/mm0506.htm).

3 C. DORNAN, op. cit., pp. 202-203.

4 W. GLlsdorf, et R. BERnier, "Pratiques journalistiques et couvertures des campagnes électorales au Canada", in Fr. FLETCHER, op. cit., p. 63. 
D'autres ont recommandé que le rôle des médias dans le processus électoral soit mieux expliqué aux citoyens, notamment en demandant aux journalistes de mieux faire connaitre les coulisses du journalisme et les tentatives de manipulation politique qui s'y déroulent ${ }^{1}$.

\section{Fondements normatifs et scientifiques de l'autorégulation}

Il me semble important d'aborder maintenant la question des fondements théoriques, normatifs et scientifiques de l'autorégulation. Cette approche ne vise pas à chasser définitivement le doute, mais bien à échapper au relativisme moral d'un postmodernisme voulant que toutes les raisons d'agir aient la même valeur morale ou soient basées sur des différences qu'il suffit d'accepter sans les discuter. $\mathrm{Si}$ l'autorégulation doit échapper à un piège, c'est bien à celui qui conduit à nier la valeur des principes fondamentaux de l'éthique et de la déontologie du journalisme : intégrité, imputabilité, impartialité, recherche rigoureuse et diffusion de la vérité, service prioritaire, voire exclusif de l'intérêt public, etc. On peut certes discuter ferme sur l'acception précise de chacun de ces termes, mais la difficulté de la tâche ne justifie pas qu'on les ignore ou qu'on puisse en nier la portée pragmatique dans les comportements professionnels quotidiens. En somme, la critique des médias ne peut pas, ne doit pas être arbitraire, impressionniste. Elle doit reposer sur de solides socles.

\subsection{L'autorégulation ne doit pas exister en dehors de fondements théoriques}

Il me semble fondamental de soutenir le postulat voulant que l'autorégulation n'échappe pas à l'exigence de se prévaloir de fondements théoriques explicites. Par exemple, la critique de certaines pratiques journalistiques -telle l'obsession des aspects stratégiques de la couverture électorale (horse race) au détriment des enjeux (issues), ou encore l'insistance accordée à la personnalité des acteurs sociaux

1 D. Hogarth et W. GILSDORF, "L'effet des services d'information continue sur les élections et leur couverture", in Fr. FLETCHER (dir.), La radiodiffusion en période électorale au Canada, Montréal, Commission royale sur la réforme électorale et le financement des partis, 1991, Vol. 21, pp. 197-198. 
importants au détriment de leurs idées ou programmes politiquesdoit s'exercer en fonction de certains postulats ou principes, qu'ils soient politiques, philosophiques ou autres. L'important ici étant d'offrir le cadre d'analyse de la critique de ces pratiques afin d'éviter les commentaires ad hoc et impressionnistes, voire corporatistes, qui caractérisent malheureusement trop souvent les jugements portant sur le journalisme.

Cela exige certes un travail intellectuel toujours exigeant, parfois douloureux, alors que notre désir premier se résume souvent à vouloir infliger une correction morale à ceux qui émettent des opinions contraires à nos croyances qui sont toujours les meilleures, c'est bien connu! Analyser et critiquer les pratiques journalistiques est un exercice dont la fécondité relève en bonne partie du système de valeurs et de la hiérarchisation de ces dernières, ce qui permet au critique de tenir compte des biais, des avantages et des limites de son poste d'observation.

Au-delà des aspects théoriques, il faut aussi prêter attention à la dimension méthodologique de l'autorégulation qui constitue sur le plan pratique un précieux gage contre les décisions arbitraires, les double standards et les jugements aberrants. La démarche adoptée pour analyser une situation et porter un jugement compte tout autant que la validité des notions théoriques retenues. Par exemple, la démarche méthodologique ne peut pas faire l'économie des règles généralement reconnues de l'argumentation valide (éviter les généralisations hâtives, se méfier des exemples atypiques, éviter les attaques ad hominem, recourir avec prudence aux appels aux conséquences, etc.). Elle doit aussi baser ses analyses et critiques sur une recherche rigoureuse des règles déontologiques, des valeurs morales et professionnelles ainsi que des principes éthiques pertinents au cas étudié, ce qui évitera les jugements aussi lapidaires qu'injustes.

\subsection{Les principaux fondements normatifs}

On l'a vu, l'autorégulation repose sur des fondements théoriques et pratiques, mais il faut aussi y adjoindre des fondements normatifs et scientifiques. Je me limiterai ici à discuter des codes de déontologie qui constituent, à mon avis, la principale source de fondements normatifs du journalisme. La question des fondements scientifiques sera abordée par la suite. 


\subsubsection{Les codes de déontologie}

.Il n'est pas inutile de rappeler les principales fonctions des codes de déontologie qui dictent les règles de conduite des journalistes à l'égard de leurs sources d'information, de leurs collègues, de leur employeur et du public qu'ils affirment servir. Les codes servent à sauvegarder la crédibilité des journalistes, protéger l'image de la profession, en valoriser le caractère professionnel, protéger le public contre les pratiques pouvant lui être nuisibles, protéger la profession contre les interventions de tiers (l'État surtout) ainsi que protéger les journalistes contre les décisions arbitraires des employeurs.

Mais il faut aussi reconnaître trois arguments soutenus par les adversaires des codes de déontologie. Il y a premièrement leur rigidité qui inhiberait le jugement personnel des journalistes en voulant s'y substituer. Deuxièmement, les codes sont souvent établis à partir de règles de conduite trop floues ou trop générales. Troisièmement, les codes pourraient être utilisés contre les journalistes et les entreprises de presse dans le cadre de procédures judiciaires. Ces arguments ne constituent pas un frein à l'élaboration de codes, mais sont des balises dont il faut tenir compte, car il existe des solutions aux deux premières objections, quant à la troisième, elle a des relents de corporatisme qu'il est toujours préférable d'éviter, les journalistes étant des justiciables au même titre que les autres acteurs sociaux.

Il faut par ailleurs avoir des attentes réalistes quant à l'impact des codes de déontologie sur les comportements réels. Leur bilan n'est pas universellement positif, certains ne contenant aucune sanction pouvant susciter l'imputabilité des journalistes, d'autres tombant dans l'oubli peu après leur adoption et on a même observé que plusieurs journalistes travaillent sans le savoir pour des médias qui possèdent un code de déontologie ${ }^{1}$. Les études ne sont par ailleurs pas catégoriques quant à l'influence des codes sur les pratiques des journalistes. Certains chercheurs ont pu observer une telle influence ${ }^{2}$ alors que d'autres ont été incapables de montrer des différences mesurables entre les journalistes soumis à de tels codes et ceux qui ne

1 D. BOEYINK, op. cit., p. 2.

2 D. ANDERson, "How managing editors view and deal with ethical issues", Journalism Quarterly, vol. 64, 1987, pp. 341-345. 
l'étaient pas, face à des cas hypothétiques ${ }^{1}$. Au terme de son étude comparative de trois quotidiens américains, Boeyink conclut qu'il faut analyser la valeur des codes autant par leur contenu que par le climat de la salle de rédaction où ils s'appliquent. Il estime que les codes ont une influence sur les pratiques dans la mesure où ils sont pris au sérieux par l'organisation de travail, où les journalistes sont encouragés à s'y référer par leurs supérieurs ou par une culture favorisant les discussions portant sur l'éthique et la déontologie professionnelle ${ }^{2}$. Les gestionnaires de médias sont pour leur part inégalement doués pour aborder ou susciter de telles discussions professionnelles, ce à quoi fait référence la distinction entre intention éthique et compétence éthique ${ }^{3}$.

La présence de codes fait toutefois la différence en matière de sanctions car plus de journalistes sont réprimandés ou mis à pied pour des transgressions à l'éthique et à la déontologie professionnelle dans les organisations possédant un code que dans celles n'en possédant pas, ce qui témoigne à nouveau de l'importance d'un environnement favorable à ces questions ${ }^{4}$. Les médias où on observe une sensibilité aux questions éthiques ont certaines des caractéristiques suivantes : les gens en autorité montrent leur attachement à des normes élevées, les entreprises ont des normes claires et articulées, les discussions à propos de sujets controversés sont encouragées dans la salle de rédaction, car les bonnes décisions émergent souvent de salles où la culture encourage les débats et les réflexions relativement à des situations controversées 5 .

\subsubsection{Les principes et valeurs en jeu}

Il ne suffit pas de se doter d'un code de déontologie. Il faut s'assurer de sa compatibilité avec les valeurs et principes professionnels qui font consensus, ce qui implique un laborieux

1 D. Prichard and M. MorgaN "Impact of ethics codes on judgments by journalists : a natural experiment", Journalism Quarterly, 1989, vol. 66, n 4, 1989, pp. 934-941.

2 D. BoeyINK, "How Effective Are Codes Of Ethics ? A Look at Three Newsrooms", in Journalism Quarterly, vol. 71, n 4, 1994, pp. 901-902.

3 J. BLACK \& R. STEele, "Professional decision making and personal ethics", Journalism Educator, vol. 46, n 3, 1991, p. 4.

4 D. BOEYINK, "Public Understanding...", op. cit., p. 3.

5 Ibid. 
travail de recherche documentaire afin de mettre à jour ces principes et valeurs. Cela permet essentiellement trois choses qui sont les suivantes : rédiger des règles déontologiques claires, expliquer leurs fondements et offrir des critères dérogatoires. Cela nous donne des codes de déontologie de seconde génération, soit ceux qui admettent que les règles déontologiques ne s'appliquent pas à toutes les situations, en tout temps. Cela permet aussi de faire face aux deux objections évoquées plus haut quant à la rigidité ou au flou des règles déontologiques.

Il faut rappeler ici l'existence de deux conditions capitales à l'existence des codes: la reconnaissance par les pairs et la représentativité des valeurs dominantes ${ }^{1}$. Il importe, par exemple, que normes professionnelles et réalité ne soient pas en conflit ${ }^{2}$. Les codes doivent refléter les valeurs des journalistes car un "principe dont il serait impossible de montrer qu'il serait délibérément et en toute connaissance de cause choisi par un groupe d'individus, n'a aucune chance d'être mis en pratique"3.

\subsubsection{La possibilité de déroger aux règles en vertu de "bonnes raisons" par opposition à la transgression arbitraire}

En plus d'énoncer des règles claires et précises, il faut les accompagner d'un guide de réflexion qui permettra aux journalistes d'évaluer, selon les situations, s'il convient de se conformer ou de déroger à la règle déontologique. En offrant un tel code, doté de critères de réflexion, on rétablit le lien entre la réflexion éthique et les règles de conduite qu'elle a générées et on lui confère la souplesse qui a souvent fait défaut par le passé.

Il faut insister ici sur le fait que les dérogations doivent être justifiables en vertu des valeurs professionnelles reconnues, ce qui exclut toute tentative de justification de transgressions en fonction d'intérêts personnels et particuliers incompatibles avec l'intérêt public, la vérité et les autres valeurs reconnues par la profession.

1 M.-Fr. Bernier, Éthique et déontologie du journalisme, Sainte-Foy, Presses de l'Université Laval, 1994, pp. 78-80.

2 W. GIEBER and W. JohnSON, "The City Hall "Beat" : a Study of Reporter and Source Roles", Journalism Quarterly, vol. 38, n³ 3, 1961, p. 296.

3 J. Couture, "La rationalité de l'éthique: théorie et pratique", Cahiers d'épistémologie, Université du Québec à Montréal, № 9106, 1991, p. 7. 
Le code de déontologie de la Society of Professional Journalists, aux États-Unis, et le Guide de déontologie de la Fédération professionnelle des journalistes du Québec, sont de bons exemples de codes souples et rigoureux, qui fournissent à la fois des règles claires et des critères de réflexion éthique qui aident les journalistes à prendre de façon rationnelle des décisions ne s'éloignant pas en principe des valeurs et principes reconnus.

Dans le cas du recours aux sources anonymes, par exemple, on reconnaît que la règle déontologique est que les journalistes doivent citer leurs sources. Le fondement de cette règle est que cela permet au public d'évaluer à la fois la crédibilité et la compétence de ces sources d'information, en plus de confirmer que les affirmations diffusées ne sont pas des commentaires déguisés du journaliste lui-même. Mais le Guide reconnaît l'existence de situations particulières et soumet les critères de réflexion suivants aux journalistes qui voudraient déroger à la règle déontologique : l'information est importante et il n'existe pas d'autres sources identifiables pour l'obtenir (dernier recours), l'information sert l'intérêt public, la source qui désire l'anonymat pourrait encourir des préjudices si son identité était dévoilée (protection contre les représailles). On ajoute que le journaliste devra expliquer les motifs pour lesquels il accorde l'anonymat et décrire la source le plus possible, sans conduire à son identification, afin que le public puisse apprécier le plus possible sa compétence, ses intérêts et sa crédibilité.

Des démarches similaires sont offertes en ce qui concerne d'autres thèmes majeurs: les procédés clandestins de cueillette d'information, les reconstitutions et mises en scène, le respect de la vie privée et les conflits d'intérêts notamment.

La démarche de recherche de fondements et d'élaboration d'un code devra être suivie de longues et parfois douloureuses délibérations au sein de la profession afin de s'assurer que le code proposé reflète réellement les valeurs de la majorité. Il faudra cependant constamment garder à l'esprit que les règles ne visent pas à apporter une caution morale à des pratiques douteuses ou inacceptables. La tentation sera parfois forte de tenter de mutiler certains principes, certaines valeurs afin d'éviter des conflits importants au sein de la profession, mais un sain débat orienté constamment sur les devoirs de la profession devrait venir à bout d'un grand nombre d'obstacles et de réticences. 
C'est au prix de la validité et de la rigueur des fondements normatifs que l'autorégulation du journalisme sera légitime et appropriée. Tenter de faire cette économie nous condamne à errer dans les limbes de l'impressionnisme, des doubles standards, des confusions plus ou moins volontaires et des indignations sélectives qui minent la légitimité et la crédibilité de la presse dans nos sociétés pluralistes mais exigeantes qui tolèrent de moins en moins de telles attitudes discriminatoires.

\subsection{Les principaux fondements scientifiques}

Outre les fondements normatifs qui insistent sur les valeurs, les principes et la réflexion éthique, l'autorégulation du journalisme doit prendre appui sur la connaissance scientifique dont les trois principaux points d'ancrage sont: la recherche sur les pratiques journalistiques, la recherche philosophique, surtout en ce qui concerne la méta-éthique qui questionne les fondements éthiques de la déontologie professionnelle et la recherche visant à mieux connaître les attentes et perceptions du public en général ou de publics particuliers (ordres professionnels, groupes sociaux, etc.).

$\mathrm{La}$ recherche scientifique relative aux pratiques journalistiques a pour objectif de mieux documenter ces dernières, mais aussi de mieux comprendre la rationalité des acteurs. Il me semble qu'une meilleure connaissance des pratiques réelles, de leurs avantages et inconvénients, enrichit le débat et assure un processus d'autorégulation plus rigoureux. La publication de recherches spécifiques au journalisme -mais aussi leur diffusion vulgarisée au besoin- alimente les débats professionnels et évite qu'ils ne se perdent dans les ornières idéologiques sans relation avec la réalité. En somme, avant de critiquer certaines pratiques et d'adjoindre les journalistes à les modifier, encore faut-il s'assurer de l'existence réelle de ces pratiques comme de leur importance. Ceci permet encore une fois de chasser le subjectivisme débridé qui contamine sérieusement bon nombre de débats professionnels. Il faut chercher à substituer l'anecdote et l'observation fortuite par l'analyse et l'observation systématique, ce que permet la méthode scientifique.

La recherche philosophique peut quant à elle prendre ses matériaux dans l'étude des valeurs et principes en jeu, ou encore dans l'élaboration de modèles de résolution de dilemmes éthiques. Mais 
elle doit aussi tenir compte des matériaux empiriques obtenus par la quête scientifique afin d'éviter de "penser à vide". Elle permet, par exemple, de poser des jugements fondés en raison quant à certaines pratiques observées, mais aussi de comparer ces dernières aux règles déontologiques en vigueur dans différentes sociétés, ou encore de suggérer de nouveaux critères de réflexion éthique qui pourraient être pris en compte par les journalistes lorsqu'ils font face à des situations exceptionnelles.

Une importante contribution de la philosophie consiste souvent à clarifier des notions fréquemment galvaudées, menant ainsi une lutte à la confusion conceptuelle qui malmène souvent la qualité des débats. C'est, par exemple, ce que Gauthier réalise au Québec quand il nous fournit un éclairage significatif sur les types d'objection soulevées face au concept d'objectivité journalistique ${ }^{1}$. Dans une perspective sociologique, afin de tenter d'atténuer la confusion qui règne en la matière, j'ai fait un travail similaire de catégorisation en ce qui concerne les différentes règles de conversation entre les journalistes et leurs sources d'information quand j'ai étudié la question des sources anonymes que l'on retrouve dans les comptes rendus journalistiques ${ }^{2}$. La qualité des mesures autorégulatrices repose aussi bien sur la connaissance empirique du journalisme que sur sa connaissance philosophique et il est tout à fait possible d'arriver à des résultats pratiques intéressants en tenant compte de ces questions théoriques que les journalistes ont trop facilement tendance à rejeter.

D'autre part, les journalistes ne doivent pas perdre le contact avec leur public, même si dans le train-train quotidien les relations avec les pairs et les sources d'information accaparent l'essentiel des interactions. La recherche permet à cet égard d'avoir régulièrement une certaine idée de la perception du public à l'égard de diverses pratiques professionnelles. Aux États-Unis, des sondages d'opinion publique sont régulièrement menés à propos de pratiques soulevant des enjeux éthiques : sources anonymes, caméras et microphones cachés, invasion de la vie privée, conflits d'intérêts. Ces enquêtes permettent de rappeler que le public a certaines attentes précises et

1 G. GAuthIER, "La mise en cause de l'objectivité journalistique", Communication, vol. 12, n 2, 1991, pp: 81-115.

2 M.-Fr. BERNIER, Quelques aspects stratégiques du recours aux sources anonymes dans les comptes rendus de certains courriéristes parlementaires de la presse écrite francophone de l'Assemblée nationale du Québec, thèse de doctorat en science politique, Université Laval, septembre 1998, 243 pages. 
qu'il est souvent opposé à de telles pratiques, ce qui conforte la légitimité de l'argumentaire de ceux qui les contestent à l'intérieur même de la profession. On peut aussi procéder par le biais d'entrevues semi-directives afin d'approfondir l'analyse.

\section{Quelques mises en garde}

Je voudrais maintenant y aller de quelques mises en garde qui visent essentiellement à faire valoir que tout mécanisme d'autorégulation doit échapper à la tentation d'une perfection totalitaire et faire en sorte que ses sanctions demeurent modérées et étroitement reliées à certaines conditions.

Ma première mise en garde est à l'effet qu'il ne faut ni chercher ni espérer obtenir un système sans faille. Cette attente imposerait, je crois, une chape de plomb sur la liberté professionnelle, notamment en imputant de façon inconsidérée un trop grand nombre de responsabilités aux journalistes. Le défi est qu'il faut à la fois accepter l'existence de failles sans chercher à les minimaliser pour autant, ce qui oblige à une vigilance constante des dérapages déontologiques dont il faudra faire l'analyse, ne serait-ce que pour en arriver à la conclusion que certains étaient imprévisibles ou d'une récurrence tellement faible qu'il est préférable de ne pas chercher à réglementer davantage.

Une seconde mise en garde est qu'il faut rechercher la rigueur de tout mécanisme d'autorégulation tout en évitant sa rigidité, ce que permettent les codes de déontologie de seconde génération dont peuvent s'inspirer les conseils de presse, les ombudsmen et les critiques des médias. Il est possible de fuir le relativisme moral sans sombrer dans les dédales logiques d'un système de pensée circulaire ou pétrifiée, coupé des fondements moraux du journalisme.

Une troisième mise en garde est qu'il ne faut pas se laisser égarer par la question à QUI aura la tâche d'évaluer les journalistes. La personnalisation de ce genre de débat est toujours stérile. Il vaut mieux l'orienter sur COMMENT 1'autorégulation sera mise en pratique, ce qui permet d'offrir un cadre général connu de tous et de critiquer en raison même de ce cadre des jugements, des décisions ou des sanctions éventuelles qui pourraient sembler manquer de rigueur ou de modération. 
Ce qui m'amène à aborder une dernière série de mises en garde reliée aux sanctions que des mécanismes d'autorégulation peuvent avoir, même si cela n'est pas toujours le cas. Il me semble que les sanctions doivent tenir compte de quatre facteurs.

Il y a d'abord les conséquences raisonnablement prévisibles de la faute commise. Il est en effet inacceptable de sanctionner un journaliste parce que ses reportages ont eu des conséquences tout à fait imprévisibles, par exemple le suicide tout à fait inattendu d'une personnalité politique mise en cause.

Il faudrait aussi tenir compte du caractère répétitif des fautes commises par le journaliste. Il peut être tout à fait justifiable de sanctionner un journaliste qui abuse de ses prérogatives et attaque à la fois la crédibilité de la profession et l'intérêt public par des comportements contraires aux normes et valeurs du journalisme.

D'autre part, toute sanction doit être relative à l'importance de la transgression des normes déontologiques et des principes éthiques en vigueur. Il y a toute une différence, je crois, entre le fait de profiter d'un survol aérien gratuit d'une zone sinistrée inaccessible par d'autres moyens et celui d'accepter des voyages gratuits aux fins de la promotion complaisante d'acteurs politiques ou économiques.

Dans le même ordre d'idée, il faut aussi tenir compte de l'importance réelle des transgressions des droits et libertés des "victimes" de pratiques journalistiques douteuses quand vient le temps de sanctionner.

\section{Conclusion}

L'autorégulation des entreprises de presse demeure en quelque sorte l'idéal journalistique, démocratique et constitutionnel, à la condition que les formes qu'elle prend soient raisonnables, rationnelles, modérées et transparentes.

Raisonnables parce que les agents qui assument cette fonction doivent demeurer conscients de la complexité des situations soumises à leur attention et porter des jugements en conséquence.

Rationnelles parce que les décisions de ces agents doivent reposer sur des règles déontologiques claires et rigoureuses, alors que la profession a souvent intérêt à adopter des règles à la limite du relativisme et de la confusion permettant de cautionner une multitude de comportements aberrants, et que les groupes d'intérêts de la société 
souhaitent parfois des règles rigides et simplistes limitant le degré d'autonomie professionnelle et facilitant la prise de sanctions à l'endroit de ceux qui les écorchent parfois.

Modérées parce que les sanctions qui découlent de l'autorégulation doivent avoir un rapport de proportionnalité avec la gravité ou la fréquence des offenses ainsi que la prévisibilité des conséquences néfastes des pratiques journalistiques en cause.

Transparentes parce qu'il s'agit là d'une valeur cardinale de l'idéologie professionnelle à laquelle adhère le public qui en fait une condition de la crédibilité et de la légitimité qu'il accorde aux journalistes.

Il faut par ailleurs tenir compte du fait que le concept d'une liberté responsable continuera toujours à alimenter les débats, tout en sachant que ce constat ne saurait constituer une raison suffisante pour chercher à éviter d'analyser et de juger les pratiques journalistiques.

Finalement, on ne doit pas ignorer que malgré la multiplication de mécanismes d'autorégulation, les publics nord-américains sont toujours mécontents et insatisfaits de leurs médias d'information. A mon avis, l'opinion publique demeurera toujours sévère à l'endroit des médias et ce en raison d'un grand nombre de motifs (idéologiques, raciaux, économiques, religieux, etc.) qui ne sont pas nécessairement reliés aux aspects éthiques et déontologiques du journalisme qui s'y pratique. Dans bien des cas, le mécontentement peut être lié à des choix éditoriaux légitimes qui expriment, par exemple, un positionnement politique contraire aux croyances de certains groupes sociaux. Il est donc tout à fait déconseillé de chercher à améliorer le bilan éthique et déontologique de la profession en espérant ainsi mettre fin aux critiques que les publics adressent aux journalistes.

Un objectif plus modeste et réaliste est simplement de prendre les moyens qui s'imposent pour faire en sorte que le public en général ait de bonnes raisons de croire que les dérapages déontologiques des journalistes ne sont pas tolérés par la profession. Cela implique notamment de multiplier les mécanismes d'autorégulation, à la condition qu'ils reposent sur des fondements normatifs et scientifiques valides, que les règles déontologiques soient rigoureuses et souples, et que les sanctions soient proportionnelles à la gravité des transgressions, ce qui évitera de laisser la critique des médias dériver sur les flots de la démagogie et des passions populaires. 